\title{
Quantitative Studies of the Internal Referencing Method with the Bulbed Capillary External Referencing Method for Proton Nuclear Magnetic Resonance Spectroscopy
}

\author{
Kozo Momoki*† and Yoshiyuki Fukazawa** \\ *10 Shirahata-higashi-cho, Kanagawa-ku, Yokohama 221, Japan \\ **Industrial Research Institute of Kanagawa Prefecture, Shouwa-machi, Yokohama 236, Japan
}

\begin{abstract}
Serious methodological defects of the internal referencing method are revealed by quantitative studies using the bulbed capillary external referencing method. Entirely new quantitative data on solute-solvent effects fatally affecting the internal referencing method are presented and discussed. Possibilities of new solute-solvent effect studies are also suggested.
\end{abstract}

Keywords Bulbed capillary external referencing method, internal referencing method, quantitative solute-solvent effect data, nuclear magnetic resonance

In the preceding paper ${ }^{1}$, a new external referencing (ex-) method using a simple reference tube device called "bulbed capillary" was proposed as giving the most accurate chemical shifts in ${ }^{1} \mathrm{H}$ nuclear magnetic resonance spectroscopy. However, most actual ${ }^{1} \mathrm{H}$ chemical shifts have been measured with the internal referencing (in-) method ${ }^{2,3}$ which has been trusted as the best method by almost all researchers. Therefore, we compared the ex-shifts ${ }^{1}$ with the usual in-shifts, as measured for the same solutes in the same sample solutions.

Fortunately, a bulbed capillary can be inserted directly into in-sample (solute+TMS binary or solute +TMS + $\mathrm{CCl}_{4}$ ternary) solutions without disturbing the in-peaks, while independently giving the reference pair peaks ${ }^{1}$ on the same spectra. Thus, in- and our ex-shifts of the same solutes in the same sample solutions can be directly obtained and compared with each other on the same spectra.

In the comparisons, the primary concern was in estimating accuracies of in-shifts which could not be made clear without a quantitative checking method. Since the ex-shifts can be obtained as almost bias-free ${ }^{1}$, any discrepancies found in the comparisons are to be ascribed almost exclusively to biases of in-shifts, from which the latter's accuracies can be estimated for the first time. By similar comparisons, accuracies of the usual ex-shifts without the diamagnetic term correction could be estimated as poor in spite of their quite high precisions. $^{1}$

The present paper reports that accuracies of the usual in-shifts could be estimated as also poor. However, the same bulbed capillary technique could give more than

† To whom correspondence should be addressed. that. By changing concentrations in the sample solutions with a bulbed capillary inserted, variations of inpeak shifts showing solute-solvent effects could also be measured easily and quantitatively.

Such quantitative studies clarify methodological defects of the in-method, which were in fact given from the beginning ${ }^{2}$ but have been ignored up to the present. Some discussion on solute-solvent effect studies with the present data will also be given.

\section{Experimental}

\section{Bulbed capillary}

Bulbed capillary and procedures for obtaining $\delta_{\text {cor }}^{0}$ values are described in the preceding paper. ${ }^{1}$ The TMS pair peaks ${ }^{1}$ when TMS exceeds about $0.2 \mathrm{~mol}$ fraction (mf) in TMS-solute binary solutions become immeasurable, because they are covered by the bulk TMS peaks approaching the pairs with increased peak heights. However, $\delta_{\mathrm{cor}}^{0}$ values can still be obtained by replacing the TMS reference ${ }^{1}$ with another reference which shows its pair peaks to be outside the bulk TMS peak shift range. Water was selected for the purpose. Thereby, the water reference gives $\left(\delta_{\text {cor }}^{0}\right)_{\text {water }}$ values with $\delta_{\text {water,sp }}=0 \mathrm{ppm}$ which is different from the original

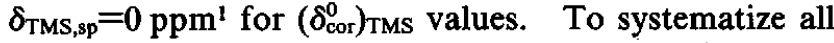
$\delta_{\text {cor }}^{0}$ values to those with $\delta_{\mathrm{TMS}, \mathrm{sp}}=0 \mathrm{ppm},\left(\delta_{\mathrm{cor}}^{0}\right)_{\text {water }}$ is converted to $\left(\delta_{\mathrm{cor}}^{0}\right)_{\mathrm{TMS}}$ by comparing these two values measured for the same solutes, as

$$
\left(\delta_{\mathrm{cor}}^{0}\right)_{\mathrm{rMS}}=1.002\left(\delta_{\mathrm{cor}}^{0}\right)_{\mathrm{water}}+4.825 .
$$

This $\left(\delta_{\text {cor }}^{0}\right)_{\mathrm{TMS}}$ is written simply as $\delta_{\text {cor }}^{0}$ and used con- 
sistently irrespective of the water or TMS reference in the following figures.

\section{NMR measurements}

NMR measurements were made with a JEOL PS-100 $(100 \mathrm{MHz}, \mathrm{CW})$ at $25.0 \pm 0.5^{\circ} \mathrm{C}$; five replicates were averaged. Careful control of the temperatures of the instrument room at $25^{\circ} \mathrm{C}$ was necessitated for obtaining the high quality data in this paper.

\section{Chemicals}

Those described in the preceding paper $^{1}$ were used throughout.

\section{Results}

\section{Accuracies of the usual in-shifts estimated}

In-shifts $\left(\delta_{\text {in }}\right)$ and our ex-shifts $\left(\delta_{\text {cor }}^{0}\right)$ for 5 typical solutes in the usual ternary in-sample solutions were measured with the above bulbed capillary technique. The results with concentrations set for the measurements are summarized in Table 1.

In Table 1, our measurements in the 6th column agree with the literature values ${ }^{2}$ in the 8 th column. However, the $\delta_{\text {in }}$ values are clearly different from $\delta_{\text {cor }}^{0}$ shifts in the 5 th column measured simultaneously on the same spectra and even far from their pure solute shifts in the 2nd column taken from the preceding paper. ${ }^{2}$

Essentially, chemical shifts are to be measured as values representing pure solutes. Therefore, accuracies of the usual in-shifts can be estimated as their biases from the pure shifts in the 2 nd column, which are unexpectedly poor $-0.3-+0.5 \mathrm{ppm}$ shown in parentheses also in the 6th column. The accuracy values are comparable to $-0.2-+0.8 \mathrm{ppm}$ estimated for the usual ex-shifts without the diamagnetic term correction. ${ }^{1}$ Thus, pre- vious in- or ex-shift values have been of qualitative nature as given poor accuracies which cannot be compared with our $\pm(0.004-0.009) \mathrm{ppm}^{1}$

\section{Solute-solvent effects affecting the in-method}

Figure 1 shows a bulbed capillary spectrum measured for a $\mathrm{TMS}(0.01 \mathrm{mf})-\mathrm{CCl}_{4}(0.99 \mathrm{mf})$ binary solution. The spectrum shows 3 TMS peaks, indicating that the bulk TMS in the solution gives a peak clearly different from the bulbed capillary TMS pair peaks. ${ }^{1}$ Thus, the $\delta_{\text {cor }}^{0}$ value of the bulk TMS peak can be evaluated readily with the TMS pair peaks.

Furthermore, the bulk TMS peaks in $\delta_{\text {cor }}^{0}$ shifts are observed as shifted downfield or upfield by increasing or decreasing $\mathrm{CCl}_{4}$ in Fig. 2, easily giving quantitative solvent effect data of $\mathrm{CCl}_{4}$ on TMS which cannot be measured with the in-method. Figure 2 prompted us to

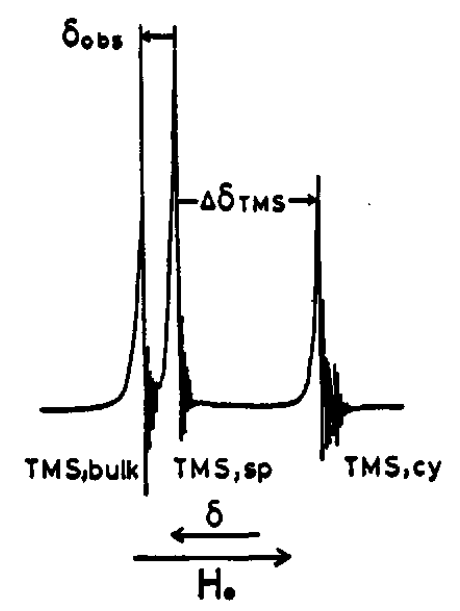

Fig. 1 Spectrum of a TMS(0.01 mf $)-\mathrm{CCl}_{4}(0.99 \mathrm{mf})$ binary solution measured with the bulbed TMS capillary device.

Table 1 Comparisons between our external referencing shifts $\left(\delta_{\text {cor }}^{\circ}\right)$ and the usual internal referencing shifts $\left(\delta_{\text {in }}\right)$

\begin{tabular}{|c|c|c|c|c|c|c|c|}
\hline \multirow{3}{*}{ Solue } & \multirow{3}{*}{$\begin{array}{c}\text { Pure solute } \\
\delta_{\mathrm{cor}}^{\circ}{ }^{\circ}\end{array}$} & \multicolumn{6}{|c|}{ Diluted with $\mathrm{CCl}_{4}$} \\
\hline & & \multicolumn{4}{|c|}{ Our measurements } & \multicolumn{2}{|c|}{ Ref. $2^{c}$} \\
\hline & & $\begin{array}{l}\text { Solute } \\
\text { vol. } \%\end{array}$ & $\begin{array}{l}\text { TMS } \\
\text { vol. } \%\end{array}$ & $\delta_{\text {cor }}^{\mathrm{a}}$ & $\delta_{\text {in }}^{b}$ & $\begin{array}{l}\text { Solute } \\
\text { vol. } \%\end{array}$ & $\delta_{\text {in }}$ \\
\hline $\mathrm{C}_{6} \mathrm{H}_{6}$ & 6.722 & 1.8 & 1.0 & $\begin{array}{r}7.348 \\
(+0.626)\end{array}$ & $\begin{array}{c}7.241 \\
(+0.519)\end{array}$ & 2 & 7.266 \\
\hline$\left(\mathrm{CH}_{3}\right)_{2} \mathrm{CO}$ & 2.042 & 3.1 & 1.0 & $\begin{array}{c}2.198 \\
(+0.156)\end{array}$ & $\begin{array}{c}2.074 \\
(+0.032)\end{array}$ & 3 & 2.085 \\
\hline $\mathrm{C}_{6} \mathrm{H}_{12}$ & 1.469 & 1.0 & 1.0 & $\begin{array}{c}1.561 \\
(+0.092)\end{array}$ & $\begin{array}{c}1.426 \\
(-0.043)\end{array}$ & 1 & 1.436 \\
\hline $\mathrm{CHCl}_{3}$ & 7.373 & 1.0 & 1.0 & $\begin{array}{c}7.349 \\
(-0.024)\end{array}$ & $\begin{array}{c}7.219 \\
(-0.154)\end{array}$ & & \\
\hline $\mathrm{CHBr}_{3}$ & 7.115 & 1.0 & 1.0 & $\begin{array}{c}6.918 \\
(-0.197)\end{array}$ & $\begin{array}{c}6.794 \\
(-0.321)\end{array}$ & & \\
\hline
\end{tabular}

+ or - value in ( ) shows the deviation of the just upper shift value from the pure solute shift given in the 2 nd column. a. Obtained from the solute peak and TMS pair peaks ${ }^{1}$ on each bulbed capillary spectrum. b. Obtained as $\delta$ distance between the solute and TMS internal reference (bulk) peaks on the same bulbed capillary spectrum as in a. c. TMS in ref. 2 was set at 1 vol.\%. 


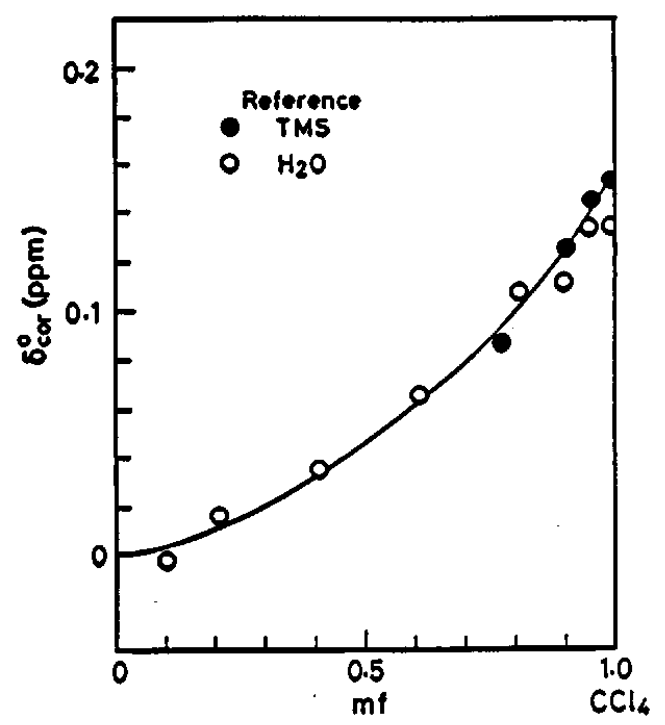

Fig. 2 Bulk TMS peak shift variation in $\mathrm{TMS}-\mathrm{CCl}_{4}$ binary system.

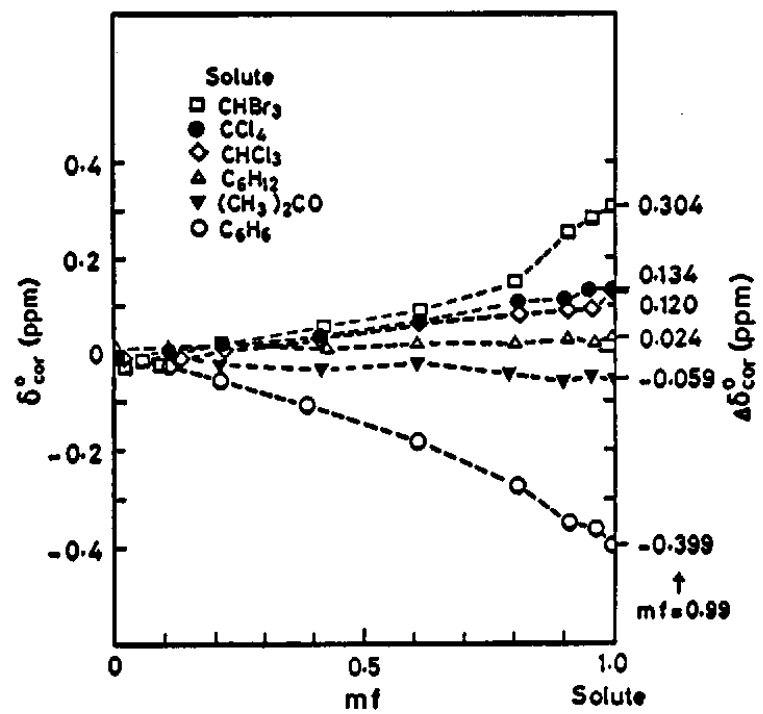

Fig. 3 Bulk TMS peak shift variations in TMS-solute binary systems.

investigate solute-solvent effects affecting the in-method further. Thus, TMS-solute (Figs. 3, 4), cyclohexanesolute (Figs. 5, 6), and $\mathrm{CCl}_{4}$-solute (Fig. 7) binary solutions were similarly measured using the same solutes as used in Table 1, where each solute giving only one ${ }^{1} \mathbf{H}$ peak was selected. Cyclohexane was taken as another reference ${ }^{4}$ for checking these TMS data.

In Fig. 3, the plottings toward the right ordinate are made up to $\delta_{\text {cor }}^{0}$ (TMS) measured at $\mathrm{mf}$ (solute) $=0.99$ as given at the ordinate. These TMS plots all converge at $\delta_{\text {cor }}^{0}=0$ on the left end at $\operatorname{mf}(T M S)=1$, revealing that the pure TMS can be represented in fact by the $\delta_{\mathrm{TMS}, \mathrm{sp}}$ reference peak as intended ${ }^{1}$ not by the usual TMS

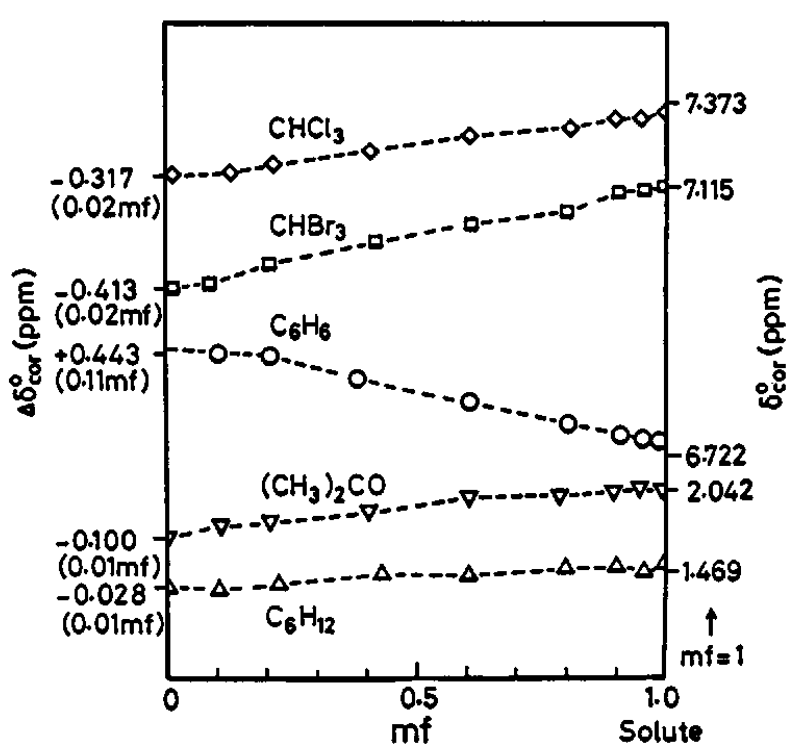

Fig. 4 Solute peak shift variations in the same TMS-solute binary systems as in Fig. 3. Figures 3 and 4 are measured simultaneously on the same spectra.

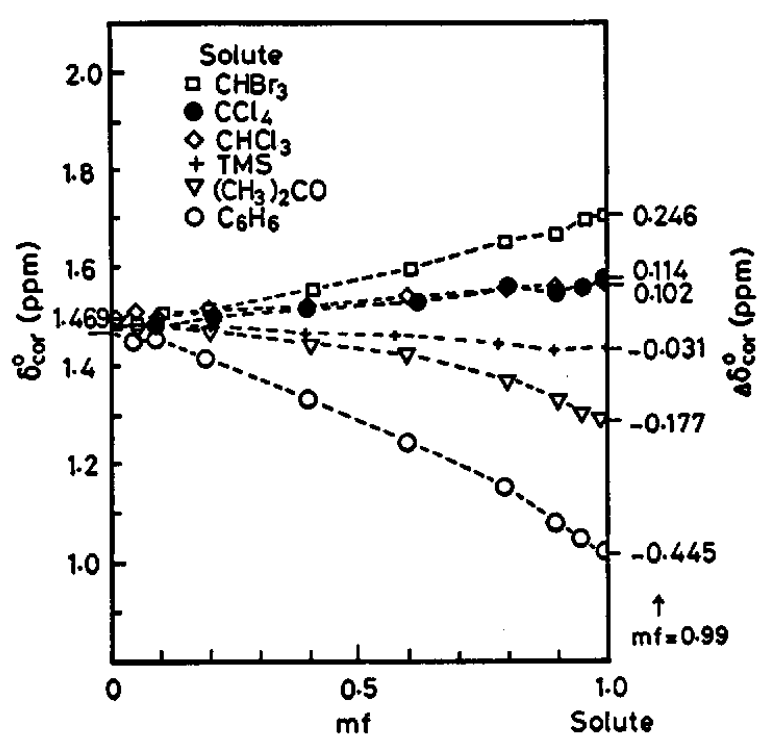

Fig. 5 Cyclohexane peak shift variations in cyclohexanesolute binary systems.

cylinder (in the ex-method) or bulk TMS (in the inmethod) reference peak. Eventually, the values given at the right ordinate in Fig. 3 represent $\Delta \delta_{\text {cor }}^{0}$ (TMS) per mf by different solutes with minus sings showing upfield shifts. Similarly, pure shifts and $\Delta \delta_{\text {cor }}^{0} / \mathrm{mf}$ with measuring $\mathrm{mf}($ solute) are given at ordinates in Figs. 4-7 for comparing each with the other. 


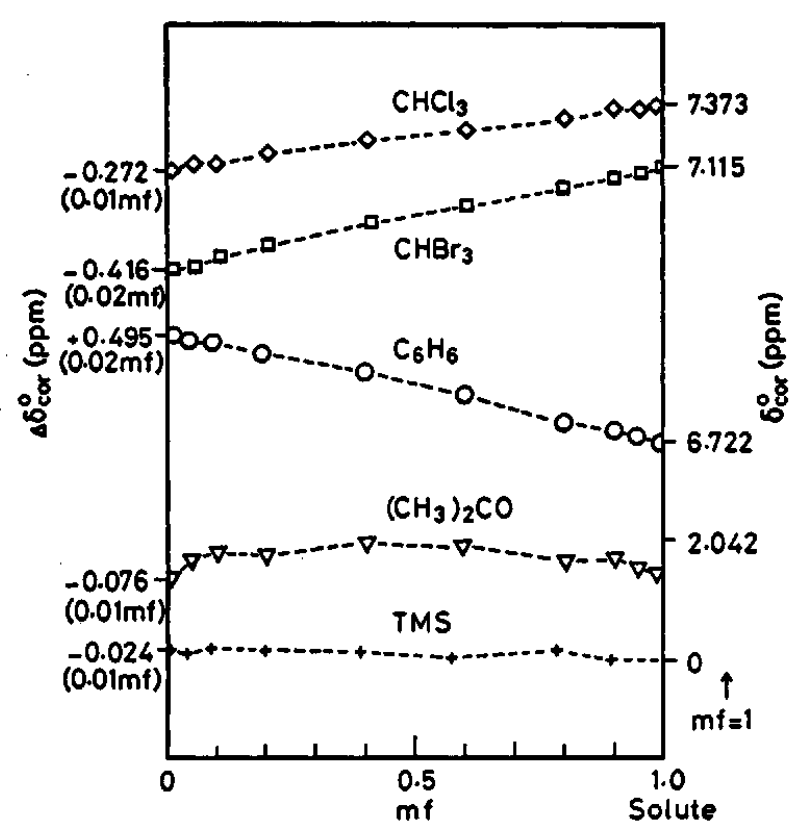

Fig. 6 Solute peak shift variations in the same cyclohexanesolute binary systems as in Fig. 5. Figures 5 and 6 are measured simultaneously on the same spectra.

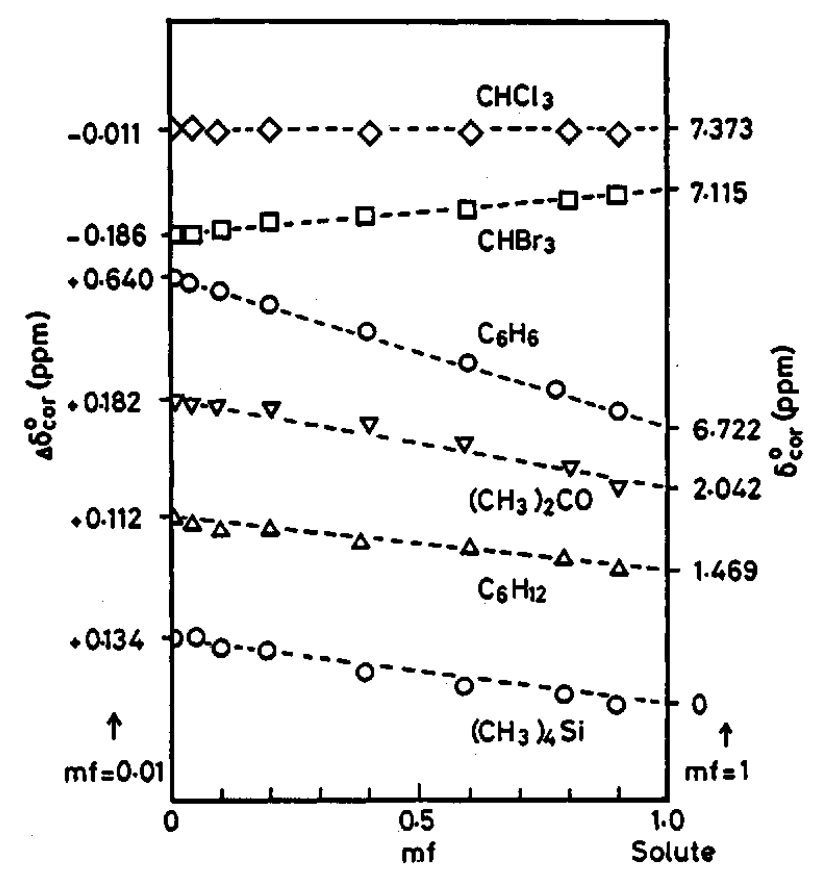

Fig. 7 Solute peak shift variations in $\mathrm{CCl}_{4}$-solute binary systems.

\section{Discussion}

Estimated poor accuracies of the usual in-shifts and solvent effects of $\mathrm{CCl}_{4}$

Since the presence of the bulbed capillary does not
Table 2 Explanation of measured ${ }^{a} \delta_{\text {cor }}^{o}$ and $\delta_{\text {in }}$ in Table 1 with different solvent effects of $\mathrm{CCl}_{4}$ on different solutes obtained $^{\mathrm{b}}$ in Fig. 7

\begin{tabular}{lcccc}
\hline \multicolumn{1}{c}{ Solute } & $\delta_{\text {cor}}^{o}$ (Fig. 7) & Dif. $^{d}$ & $\delta_{\text {in }}$ (Fig. 7) & Dif. $^{\text {f }}$ \\
\hline $\mathrm{C}_{6} \mathrm{H}_{6}$ & 7.362 & 0.014 & 7.228 & 0.013 \\
$\left(\mathrm{CH}_{3}\right)_{2} \mathrm{CO}$ & 2.224 & 0.026 & 2.090 & 0.016 \\
$\mathrm{C}_{6} \mathrm{H}_{12}$ & 1.581 & 0.020 & 1.426 & 0.021 \\
$\mathrm{CHCl}_{3}$ & 7.362 & 0.013 & 7.228 & 0.009 \\
$\mathrm{CHBr}_{3}$ & 6.929 & 0.011 & 6.795 & 0.001 \\
\hline
\end{tabular}

a. For solute+TMS+CCl 4 ternary sample solutions.

b. For solute $+\mathrm{CCl}_{4}$ binary sample solutions.

c. Taken at $\mathrm{mf}\left(\mathrm{CCl}_{4}\right)=0.99$ in Fig. 7.

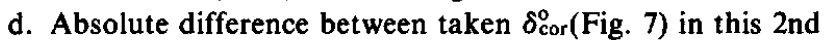
column and measured $\delta_{\text {cor }}^{\circ}$ in the 5 th column of Table 1 .

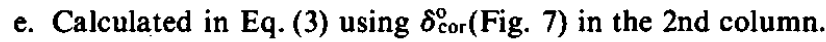

f. Absolute difference between calculated $\delta_{\text {in }}$ (Fig. 7) in this 4th column and measured $\delta_{\text {in }}$ in the 6th column of Table 1.

disturb solute and TMS reference peaks, $\delta$ in shifts in Table 1 are measured as $\delta$ distances between the two peaks directly on the bulbed capillary spectra but written also in $\delta_{\text {cor }}^{\circ}$, as

$$
\delta_{\text {in }}(\text { solute })=\delta_{\text {cor }}^{0}(\text { solute })-\delta_{\text {cor }}^{0}(\text { TMS }) .
$$

However, the $\delta_{\text {cor }}^{0}$ (solute) and $\delta_{\text {cor }}^{0}$ (TMS) are suggested in Figs. 3-7 as always shifted independently and more or less differently by the sample solutions. Therein, although Table 1 is measured for ternary sample solutions while Figs. $3-7$ are for binary solutions, $\mathrm{CCl}_{4}$ occupying 96-98 vol.\% of the ternary solutions in Table 1 was especially noted in showing considerable solvent effect differently on different solutes in Fig. 7.

Thus, although measured shifts in Table 1 may not be affected only by solvent effects of $\mathrm{CCl}_{4}$, the former was assumed as affected solely by the latter to nearly the same amounts as those obtained in Fig. 7. Therefore, $\delta_{\text {in }}$ (Fig. 7) to be at $\mathrm{mf}\left(\mathrm{CCl}_{4}\right)=0.99$ were calculated for each solute using data in Fig. 7, as

$$
\begin{aligned}
\delta_{\text {in }}(\text { Fig. 7) } & =\delta_{\text {cor }}^{0} \text { (pure) }+\Delta \delta_{\text {cor }}^{0} \text { Fig. 7) }-\delta_{\text {cor }}^{0}(\text { TMS }) \\
& =\delta_{\text {cor }}^{0}(\text { Fig. 7) }-0.134,
\end{aligned}
$$

where $\delta_{\text {cor }}^{0}\left(\right.$ Fig. 7) refers to $\delta_{\text {cor }}^{0}($ solute $)$ at $\mathrm{mf}\left(\mathrm{CCl}_{4}\right)=0.99$ measured in Fig. 7. Although actual $\delta_{\text {cor }}^{0}$ (TMS) values measured for Eq. (2) in Table 1 were variable as $0.124 \pm 0.011 \mathrm{ppm}$ for these different solutes, an identical $0.134 \mathrm{ppm}$ at $\mathrm{mf}\left(\mathrm{CCl}_{4}\right) \pm 0.99$ in Fig. 7 is also assumed in Eq. (3) as $\delta_{\text {cor }}^{0}(T M S)$ for all the same solutes.

$\delta_{\text {cor }}^{0}$ (Fig. 7) taken for Eq. (3) from Fig. 7 and $\delta_{\text {in }}$ (Fig. 7) calculated in Eq. (3) are shown in Table 2, where excellent agreements shown in the 3rd or 5th column between values deduced from Fig. 7 and those measured in Table 1 for either $\delta_{\text {cor }}^{0}$ or $\delta_{\text {in }}$ are surprisingly obtained. Thus, the poor accuracies of the usual in-shifts estimated in Table 1 could be explained quantitatively as based predominantly on different solvent effects of $\mathrm{CCl}_{4}$ on 
different solutes inevitably caused in the sample solutions.

Table 2 is surprising also in that $\delta_{\text {cor }}^{0}$ values independently measured for pure solutes before ${ }^{1}$, binary solutions in Fig. 7, and ternary solutions in Table 1 can be quantitatively connected. Such high quantitativities of measured chemical shift values cannot bex expected without the bulbed capillary ex-shifts.

\section{Methodological defects of the in-method}

Although ${ }^{1} \mathrm{H}$ chemical shifts have been measured mostly with the in-method, its bases have been not so clear or sound in fact from the beginning. ${ }^{2}$

TMS was first proposed" as "a particularly suitable internal reference" because of "magnetically isotropic, non-associative, and chemically unreactive". "In-shifts so measured in $\mathrm{CCl}_{4}$ solution are highly reproducible, are commonly independent of temperature or concentration and agree closely with the most precise and reliable published ex-measurements obtained by extrapolation to infinite dilution in $\mathrm{CCl}_{4}$ " was added ${ }^{2}$ with rough data of qualitative nature inevitable at that time.

$\mathrm{CCl}_{4}$ as a dilution solvent was proposed ${ }^{5}$ just earlier thian this, when ex-shifts of methyl halides showing absurd shift orders were first found at infinite dilution in $\mathrm{CCl}_{4}$ to be linearly related to the electronegativities of the halogens. "At the start of this work, Purcell suggested that the use of 'spherical' solvents might eliminate contributions due to magnetic anisotropy. The magnetic effects of the magnetically isotropic and somewhat 'spherical' solvents, $\mathrm{CCl}_{4}, \mathrm{SF}_{6}$ and $\mathrm{C}\left(\mathrm{CH}_{3}\right)_{4}$, are averaged to zero with time" was commented ${ }^{5}$ also with qualitative data.

The above "suitable reference" seemed to imply that the reference peaks would not be shifted in mixture sample solutions. Thus, cyclohexane or TMS as an "inert" internal reference was checked on this point ${ }^{4}$ to find least shifts of the peaks. However, the check ${ }^{4}$ had to be made with benzene as a reference for qualitative exmeasurements. No quantitative checks of such "inert" references or dilution solvents seem to have been made due to lack of a suitable checking method.

Nevertheless, the usual in-method using TMS and $\mathrm{CCl}_{4}$ has been most preferred probably because of its ease, since another ex-method has been given an erroneous impression" ${ }^{1}$ of being "not an easy technique". ${ }^{3}$ Still, chemists have sometimes feared that $\mathrm{CCl}_{4}$ might cause solvent effects on solutes because of its halogens. Probably due to this fear, "the disadvantages (by solvent effects) of the in-method may be minimized (to be made negligibly small); if a number of solutes are investigated in the same solvent $\left(\mathrm{CCl}_{4}\right)$ and with the same internal reference (TMS), all the solvent effects so obtained are in error (but) by the same (small) constant amount (to be neglected)" was described. ${ }^{3}$ The current wide usage of the in-method seems to be based on this comment. However, since no quantitative verifications of "by the same constant amount" have been possible, the comment seems to be nothing but one expressing intuitive expectations for the easy in-method.

The present paper gives experimental data against such expectations. Even if solutes are measured with the same TMS and $\mathrm{CCl}_{4}$, considerable solvent effects of $\mathrm{CCl}_{4}$ which differ on different solutes including TMS (Fig. 7) are always caused in the sample solutions to give inevitably poor accuracies for the usual in-shifts (Tables 1,2). Even the reference TMS or cyclohexane peaks in mixture solutions are not invariable, but are always shifted more or less differently from sample to sample due to inevitable solvent effects (Figs. 3, 5). Also due to solvent effects, solute peaks in mixture solutions are always shifted independently and more or less differently by the samples (Figs. 4, 6).

Eventually, in-shifts needing to be measured in mixture sample solutions cannot represent the pure solutes because of such inevitable solvent effects. Against the usual understanding, infinite dilution in $\mathrm{CCl}_{4}{ }^{2,4,5}$ does not improve but rather makes the situation worse. Thus, serious methodological defects of the inmethod in fact given from the beginning ${ }^{2}$ but ignored are now shown to be primarily in necessitating mixture sample solutions at least by the use of internal reference.

Because of inevitable solvent effects, the usual in-shifts are at best given poor accuracies as mentioned, but sometimes even provide erroneous information on solutes: in Table 1 , benzene comes at $0.45 \mathrm{ppm}$ more downfield than bromoform in their in-shifts (6th column), whereas reversely at $0.4 \mathrm{ppm}$ more upfield in the pure solute shifts (2nd column). This reversal can be explained quantitatively also with different solvent effects of $\mathrm{CCl}_{4}$ on each species (Fig. 7, Table 2).

Therefore, in-shifts cannot be defined without the sample compositions.

\section{Some preliminary discussion on solute-solvent effect studies with our new quantitative data}

In-shifts seem disadvantageous also for solute-solvent effect studies in other points in addition to their qualitative or erroneous nature discussed above.

In every binary system in Figs. 3-6, solute and reference peaks are simultaneously shifted by nearly the same amount toward the same direction at varied $\mathrm{mf}$. Therefore, in comparing variations of $\delta_{\text {in }}$ and $\delta_{\text {cor }}^{0}$ for 3 typical solutes in Fig. $8, \delta_{\text {cor }}^{0}$ shifts forming more inclined plots a) are leveled off in $\delta_{\text {in }}$ plots b) or c) by subtractions in Eq. (2), though the levelings-off are made almost completely for benzene, while incompletely for chloroform or bromoform. Such levelings-off typically for benzene might lead to "in-shifts are independent of concentration" as their advantage. ${ }^{2}$

However, levelings-off in in-shifts must be disadvantageous for solute-solvent studies, since some effects may be concealed in the levelings-off. Especially, complex-formation ${ }^{6-8}$ or self-association ${ }^{9-11}$ theories usually using such leveled-off data of inaccurate in-shifts for necessary curve-fitting which in principle need accurate data are quite questionable. Probably from this reason, $K=10^{9.5}$ was once reported for a TMS- 


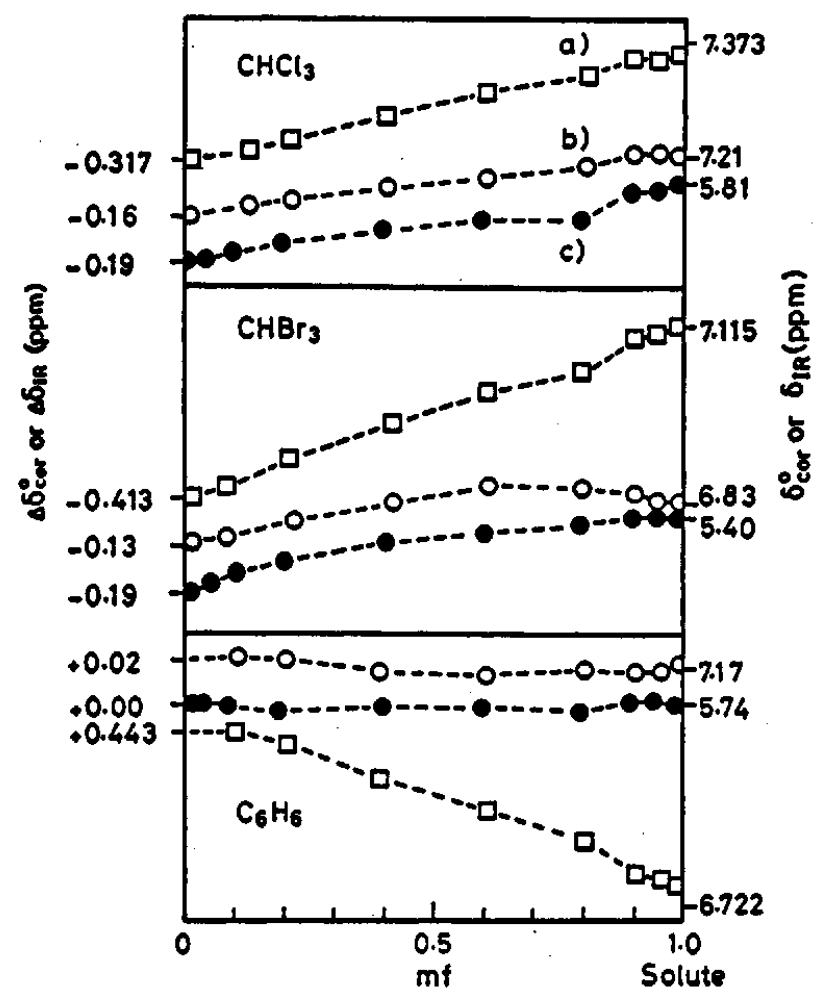

Fig. 8 Comparisons between bulbed capillary $\delta_{\text {cor }}^{\circ}$ and internal referencing $\delta_{\text {in }}$ shifts on the same binary sample spectra. a), $\delta_{\text {cor }}^{\circ}$ shift; b), $\delta_{\text {in }}$ shift with TMS as the reference; c), $\delta_{\text {in }}$ shift with cyclohexane as the reference. Values given at the ordinates for $\delta_{\text {cor }}^{0}$ are taken from Fig. 4 , while those for $\delta_{\text {in }}$ are values by extrapolations to each ordinate using measured ones in the vicinities.

benzene complex-formation. ${ }^{8}$

Each plotted curve in Fig. 3, if measured separately, may be studied for a complex-formation or selfassociation. However, complexed (TMS) ${ }_{m} \mathrm{X}_{n}$ or selfassociated (TMS) $)_{m}$ species can hardly be considered systematically in Fig. 3 for such a series of curves each of which differs more or less from the other not only in shift magnitude but sometimes even in shift direction. Especially, shift directions are to be no less impotant than shift magnitudes, where relative $\delta_{\text {in }}$ cannot directly give shift directions but magnitudes in contrast to $\delta_{\text {cor }}^{0}$ which can give the both. Thus, results of the above theories as in the above absurd $K$ value are questionable as based only on shift magnitudes by $\delta_{\text {in }}$ data. Also, a pair of solute and reference peaks gives two independent $\delta_{\text {cor }}^{0}$ data against only one $\delta_{\text {in }}$, while even systems such as TMS- $\mathrm{CCl}_{4}$ cannot be measured in $\delta_{\text {in }}$ shifts but only in $\delta_{\text {cor }}^{0}$ shifts.

Therefore, not one or two curves but many curves possibly in Figs. 3-7 are better considered collectively and systematically for solute-solvent effects by taking shift magnitudes and directions into account. For the purpose, $\delta_{\text {cor }}^{0}$ data which can be compared quantitatively between independent samples or measurements (Table 2) must be indispensable. Thus, Figs. 3-6 can be understood as follows. A "solution" made by dissolving a solute in a solvent always implies that some mutual interactions between the solute and solvent species necessarily exist. The mutual interactions will naturally give rise to simultaneous solute and solvent effects in causing both solute and solvent shifts at the same time for the system, as in Figs. 3-6. The necessity of such mutual interactions has long been realized but quantitative data directly showing the fact have been scarce. Figures 3-6 suggest the first such NMR data.

Some fundamental points can now be derived:

i) Mutual interactions must be characteristic not of a solute or solvent alone but only of the solute-solvent combinations. Therefore, plotted curves of resulting solute-solvent effects on each peak must also be characteristic only of the solute-solvent combinations, not of a solute or solvent alone, as Figs. 3-6 show. Curves in Fig. 7 are naturally different for different solutes.

ii) Each point on these curves must be considered as simultaneously affected by all species including the peak species in the sample solution. The effects of all species may work additively or subtractively, depending on their directions, again suggesting the importance of direction studies expected only with $\delta_{\text {cor }}^{0}$ data. Effects of some species may be neglected as small compared with those of one occupying most of the sample solution or showing particularly large effects, leaving the effects of the particular species as predominant. Solvent effects of $\mathrm{CCl}_{4}$ in Fig. 7 and Table 2 may be this case.

iii) TMS or cyclohexane as an "inert" internal reference $e^{4}$ is finally checked quantitatively. As mentioned, TMS was initially chosen because of "chemically unreactive", 2 probably implying that TMS would be indifferent to solute-solvent effects. No effects of others on TMS or cyclohexane have been thought as assured but only with qualitative checks ${ }^{4}$, while no effects of TMS or cyclohexane on others seem to have been taken as rather natural without checks.

In our quantitative data, effects of others on TMS (Fig. 3) or cyclohexane (Fig. 5) are slight but cannot be neglected (Table 2). Effects of TMS (Fig. 4) or cyclohexane (Fig. 6) on others appear to be appreciable. However, in the figures, both the TMS and cyclohexane peaks are almost not shifted in TMS-cyclohexane system. Thus, effects of TMS or cyclohexane on others may be very smáll due to its nature and Fig. 4 or 6 must predominantly show variations of solute effects on the same solute peaks themselves, as discussed in ii).

Therefore, TMS or cyclohexane may be "inert" only in their effects on others. The initial "inert"4 for no effects of others on TMS or cyclohexane is not true quantitatively.

iv) The above discussion especially on Figs. 4 and 6 suggests that $\delta_{\text {cor }}^{0}$ plots by varying mf may be studied better by microscopically considering mutual interactions between the species and its surroundings in the sample solutions. Thereby, intermolecular effects which are expected to be varied by $\mathrm{mf}$ become more 
attractive than intramolecular effects which must be least varied by $\mathrm{mf}$. Solute effects on the same solutes themselves expected in Figs. 4 and 6 may be conceived best by considering intermolecular interactions between the same solutes to be at $\mathrm{mf}$ (solute) very close to 1 . Not only shift magnitudes but also shift directions should always be carefully observed and considered.

Some $\delta_{\text {cor }}^{\circ}$ plots in Figs. 3-6 will be explained systematically by considering intermolecular effects in addition to intramolecular effects. By connecting and comparing $\delta_{\text {cor }}^{0}$ data for selected samples, some of these effects can be evaluated even numerically, as will be reported. Thus, $\delta_{\text {cor }}^{0}$ data will become valuable for solute-solvent effect studies.

\section{References}

1. K. Momoki and Y. Fukazawa, Anal. Chem., 62, 1665 (1990).

2. G. V. D. Tiers, J. Phys. Chem., 62, 1151 (1958).
3. F. H. A. Rumens, "Van der Waals forces in NMR intermolecular shielding effects (NMR basic principles and progress Vol. 10)", Chap. 15, pp. 98-105, Springer-Verlag, Berlin, 1975.

4. E. D. Becker, J. Phys. Chem., 63, 1379 (1959).

5. A. L. Allred and E. G. Rochow, J. Am. Chem. Soc., 79, 5361 (1957).

6. C. M. Huggins, G. C. Pimentel and J. N. Schoolery, J. Chem. Phys., 23, 1244 (1955).

7. C. J. Creswell and A. L. Allred, J. Phys. Chem., 66, 1469 (1962).

8. J. Homer, E. J. Hartland and C. J. Jackson, J. Chem. Soc., 931 (1970).

9. C. M. Huggins, G. C. Plimentel and J. N. Schoolery, $J$. Phys. Chem., 60, 1311(1956).

10. L. W. Reeves and W. G. Schneider, Can. J. Chem., 35, 251 (1957).

11. C. F. Jumper, M. T. Emerson and B. B. Howard, J. Chem. Phys., 35, 1911 (1961).

(Received November 8, 1990) (Accepted April 1, 1991) 